\title{
Mobile Apps for Learning Argumentation Skills: Exploring Student Perception of Dialog Games on Tablet Devices
}

\author{
Ilker Yengin and Bojan Lazarevic
}

\begin{abstract}
To meet the challenges of the 21 st century, it is essential for students to master advanced communication skills that are required for increasing innovation and collaboration performances in their future workplaces. Dialog games are one of the proven teaching-learning methodologies which improve communication and collaboration skills. The benefits and effects of using dialog games in the learning setting are well studied. However, there is no study which focuses on the possibility of using dialog games on mobile devices. Considering the growing trend of mobile application use in learning, it is important to understand students' attitudes towards the possible use of dialog games in mobile learning settings.

In order to address such a need, this paper discusses the design of mobile applications by way of using dialog games methodology as well as the results of a mixed method study that investigates the usability of the mobile application and students' attitudes toward it. For the purpose of this study, we conducted a usability test and an interview. The analysis shows that students have positive attitudes toward dialog games on tablet devices and that they perceived benefits (e.g. suitable for school, facilitate learning) of dialog games for learning (e.g. enabling social interaction, guidance to focus on thinking, critical and creative thinking).
\end{abstract}

Index Terms-Dialog games, m-learning, human computer interaction, educational technology.

\section{INTRODUCTION}

Learning and innovation are a key component addressed in the Horizon 2020- The EU Framework for Programme for Research and Innovation [1]. Similarly, Framework for 21 st Century Learning puts a strong emphasis on the development of learning and innovation skills [2]. According to this framework, to successfully develop learning and innovation skills, it is essential to be able to establish clear communication in collaborative learning activities.

As the framework suggests, it is a requirement to provide a support system to help students master the abilities that will be required for managing the technical and global challenges in the decades to come. Using dialog and argumentation for teaching and learning is one of the proven methodologies designed to support students' communication in collaborative learning activities [3]. Dialog games are tools which are known to facilitate the construction of structured communication behavioral patterns between learners, such as

Manuscript received March 3, 2017; revised May 12, 2017.

Ilker Yengin is with Institute of High Performance Computing, A*STAR, Singapore (e-mail: yengini@ihpc.a-star.edu.sg).

Bojan Lazarevic is with Mount Saint Mary College, New York, USA (e-mail:Bojan.Lazarevic@msmc.edu, lazaemail@gmail.com). assistance, information-seeking, probing, and peer teaching [4]-[7].

Expecting an exponential growth in using mobile applications in the future [8], it may be significant to understand students' attitudes toward the use of dialog games in the mobile learning setting. Although the use of dialog games in education has been investigated in previous research studies [9], there is no study investigating their use in mobile learning applications, especially on tablet devices.

To address this need, this paper discusses the design of dialog games on tablet devices, the usability of their design and students' attitudes toward this learning methodology. The purpose of this mixed method study is to provide an in-depth understanding of the users' attitude towards the proposed design of dialog games. For this objective, a usability study and an interview were conducted. The analysis shows that students have positive attitudes toward the dialog game on tablet devices and that they understand its benefits for learning.

This paper is beneficial for in-service teachers and education policy makers, i.e. administrators, who seek to understand teaching argumentation skills using mobile (tablet) devices as well as mobile apps developers interested in the dynamics of dialog games for tablet devices.

\section{Dialog Games IN LEARNING}

Contemporary versions of dialog games are considered as educational tools (usually online) enabling students to put forward their arguments in a discussion by providing a platform for logical and structured cognitive patterns in dialogic interactions between the participants [10], [11].

Dialog games use methods which help participants to indicate their communicative acts (e.g. "agree," "challenge,") more clearly, which differs from regular day-to-day dialogic interactions. This enables the facilitation and structuring of effective learning interactions which occur within interactive dialogs. The large body of literature informs that learning scenarios which incorporate dialog games as part of educational process improve students' learning and advance their argumentation and reasoning skills [12], [13].

Dialog games, as a method for engaging students in collaborative interactions, may help learners develop advanced argumentation skills through fostering well-structured interactions. Furthermore, empirical evidence supports the general instructional value and effectiveness of socio-cognitive applications of dialog games in educational settings [14]. 
Dialog games tools are designed to improve collaborative dialogs and highlight effective goals and moves in collaborative learning interactions. Ravenscroft [15] emphasizes the effectiveness of dialog games applications compared with other online educational tools designed for communication such as chats or synchronous discussion boards. The unique effectiveness of dialog games is a result of the specific design of their interaction platform, which is aimed at promoting high quality and engaging critical discussions which bring positive changes in cognitive reasoning. Other platforms and traditional online education tools such as chat and threaded discussion have several downsides such as possibly allowing a few participants to dominate in the discussion, which can have a negative impact on learning.

Recent studies in learning sciences strongly emphasize the importance of collaboration and critical thinking in educational environments [16], [17]. These two crucial areas have also been emphasized in recent advances in $21 \mathrm{st}$ Century Learning movement [18]. As a reflection of this trend, curricula developers across a variety of academic fields encourage the integration of collaborative learning and critical thinking components in different phases of instruction. Teachers, researchers and practitioners in the field of education are now facing a new challenge - to implement effective teaching methods, and to foster students' collaboration and critical thinking [19].

\section{Design of Dialog Game Mobile Application}

Employing dialog-based learning interactions toward these ends is a potentially effective strategy. Dialog-based learning interactions enable students to understand both their own and others' differing perspectives, a critical element of both collaborative learning and critical thinking [20]. These metacognitive skills are central to communications that build positive social relationships and effective collaborative learning environments [21]-[23]. Learning interactions through dialogs also improves cognitive flexibility in terms of learners modifying their thinking styles and adopting others' mental models [24].

Expanding and transforming the available dialog game applications in the literature, the research team has developed a new dialog game prototype which can run on mobile devices. The current version of the prototype is designed for tablet devices due to the availability of bigger screens, which makes reading and typing easier. All usability tests carried out in this study are valid for the tablet version of the prototype.

The prototype can also be used on smart phones including both iOS and Android due to the cross-platform flexibility of coding language performed in the development process. The coding language used in the development is Live Code [25]. Live Code is a language that enables developers to create apps for multiple platforms.

Using Live Code in the development allowed us to utilize the same code for different mobile platforms so as to make them accessible to a greater number of students with reduced effort. Although this enabled the research team to run the dialog game app for iOS and Android platform, usability studies were carried out on the iOS platform due to its availability.

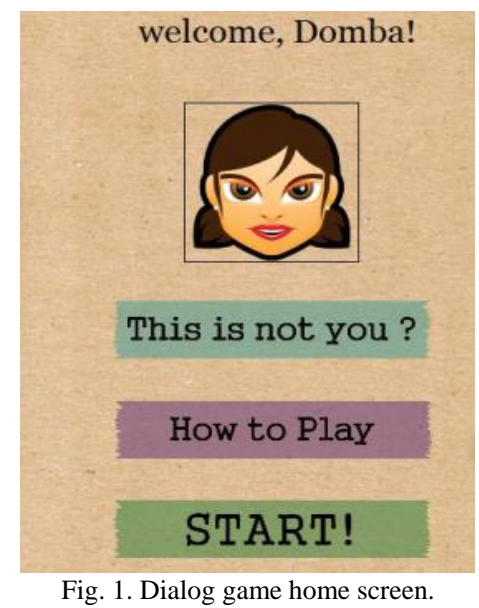

\section{A. User Interactions}

Fig. 1 and 2 represent the prototype mobile app design for the prototype dialog game screens. In the top left corner of the main dialog screen there are three buttons (Fig. 2 - A) which allow students to return to the home screen (player settings and discussion room entry screen), saving current dialog into a file (.pdf) and printing the file saved. The top text field (Fig. 2 - B) shows the main topic of the dialog. The main topic usually contains an idea or question which serve as the seed topic of the dialog. The topic can be set by the teacher or by students before the dialog.

Although it is possible to modify it for multi-player interaction, the current prototype version is designed for dialogs between only two players for the purposes of this research. The current prototype version allows users to play dialog games by turn taking. Turn-taking strategy in the dialog game is applied so as to allow the dialog flow to remain coherent and logical.

Turn-taking in the interactions allows participants in dialogs to closely observe and follow the developing dialog at their own pace. Dialogs are given in the linear format to make the display more coherent. In Fig. 2, the main window (Fig. 2 - C) lists the dialog flow including turn numbers, icons of the participants, as well as the moves they initiated.

Building upon the original dialog game idea of Ravenscroft and collaborators [14]-[18], the current design requires the participant to select the moves (or communicative acts) among the items that are listed as colorful buttons under the main dialog flow form (Fig. 2 - D). Currently, there are six different moves defined by the following categories: "Inform", "Question" "Challenge", "Reason", "Agree" and "Maintain". After selecting the moves, players need to select specific sentence openers from the left side list menu in order to begin to construct their expressions (Fig. 2 - E).

The interaction mechanism for constructing sentences is designed in accordance with the suggestions given by the current literature on dialog games. Current literature confirms that these types of moves and sentence openers are designed to help participants focus on structuring their cognitive patterns in any ongoing dialog [15], [19]. 
Dialog partners are pushed to pay attention to the sentence openers in order to use well structured dialog actions which are modeled according to communicative acts referenced in a large body of research literature [11]. Using modeled and predetermined communicative acts and cues in dialog games encourages dialog participants to strategically use dialog game moves to structure their flow of thinking and consider the patterns of the ongoing dialog.

Sentence openers as dialog strategies also force the participants of the dialog to keep their focus on the dialog and to think carefully before submitting their responses. The strategies are designed to facilitate dialog actions, such as questioning and qualifying propositions, introducing evidence and prompting rebuttals.

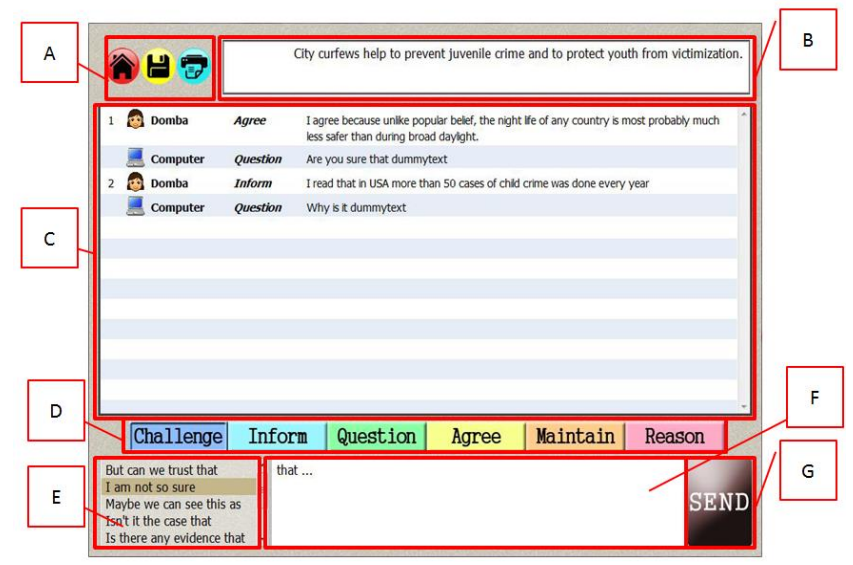

Fig. 2. Main dialog game screen.

According to the design of the dialog game app screen, a basic user interaction flow scenario for a typical player of the dialog game is as follows (assuming that the player name, icon, login and dialog topic are already set):

1) Player starts the game by clicking the Start button (Fig. 1).

2) Once it is his/her turn, player should respond to an idea. The idea can be either the initial seed idea (Fig. 2 - B) or an idea proposed by other players (Fig. $2-\mathrm{C}$ ).

3) Player selects a dialog move by clicking one of the moves listed (Fig. 2 - D).

4) Player selects an appropriate opener to start his/her sentences (Fig. 2 - E).

5) Player expresses his/her ideas and responses by filling in the free-text form (Fig. 2-F) to continue the sentence started with the openers in the previous step.

6) Player clicks "SEND" button to complete his/her move (Fig. 2 - G).

\section{Methodology}

The aim of this study is to shed light on students' attitudes toward using dialog games on mobile platforms. For this purpose, a prototype mobile dialog game app was designed as described in the above section. In order to understand participants' attitudes toward dialog games, this study applied a mixed method research which includes a usability study and a post-interview with the participants.

The purpose of the first phase of the usability study was to identify usability issues in the application and to look into participants' capabilities to complete the tasks in the dialog game. In the second phase, a structured interview was conducted immediately after the participants completed the dialog game.

Participants of the study were selected using a purposeful sampling method that uses filtering criteria which include the following: participants had no prior experience with dialog games, but were able to use the computer and/or mobile tablet devices. The total number of the participants is 20 with an average age of 18 .

The usability test method collects both quantitative and qualitative data sets and the interview method collects qualitative data sets. In the usability test, the interactions with the dialog game application were video recorded. In the interview, participants' responses were audio recorded. The researchers reviewed the recorded user interactions and interview responses and encoded them into text files and visual interaction maps (see Fig. 3).

\section{A. Data Analysis}

Data analysis of this study is based on a mixed method approach. Participants' interactions were encoded into visual interaction maps. The interaction maps were analyzed employing a frame analysis, while the interview data was analyzed using coding techniques for an interpretation analysis, as described by Creswell [26], [27].

For data reliability, following Gibbs' strategies [28], trained researchers verified a random sample of data. Identified codes were double-checked for drifts. For data validity, data sources were triangulated. Considering that the researchers used direct measures, the applied protocol of the usability test is valid.

\section{B. User Interactions Analysis}

The user interaction analysis phase of the study measures user response time while executing a particular common user scenario including different task sequences in the designed dialog game mobile app.

For quantitative usability data analysis, every interaction of the participants in the dialog game was recorded and the average time for each move was calculated in Table I.

TABLE I: AVERAGE TASK EXECUTION TIMES

\begin{tabular}{|l|l|l|}
\hline Symbol Type & Meaning & Average Timing (s) \\
\hline & Change Name & 5 \\
\hline \\
\hline
\end{tabular}

Table I shows the possible user interaction in a particular use case scenario. User interactions and actions are represented by symbols and labels in the table with the 
corresponding meanings and average response time next to them. Calculations of average response times are retrieved from the captured video that shows users' interaction in the dialog game mobile app. Table I was constructed on the basis of the following scenario:

"A student signs in the system and joins a discussion topic by typing his/her name and choosing an avatar icon. Student may directly start the game or read the instructions about the dialog game play. Then students start playing the dialog game by replying to the initial seed idea. The game ends after 30 turns."

In the above scenario, students were partnered with a research staff member who also took part in the dialog game. The responses given to the students were provided in a timely feedback fashion, thus, the waiting time for the research staff responses was not included in the calculations. Dialogs ran only between student and researcher (as teacher or computer).

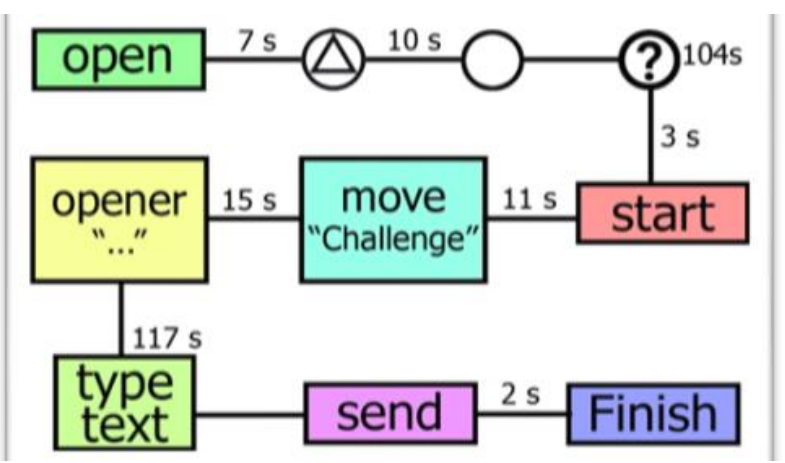

Fig. 3. Example of user interaction task flow map.

In addition to the calculation of average user responses, each task and interaction executed by the users are encoded into user interaction flow maps to provide an overall view. These maps show individual performances in terms of task completion time.

Fig. 3 is an example of user interaction task flow map. According to example in Fig. 3, the particular user mostly spent time on reading "how to play instructions" (104s) and typing his/her response $(117 \mathrm{~s})$ in the free-text form (Fig. 2-F).

\section{User Interviews}

User interviews are the second component of data analysis. In this phase the researchers relied on qualitative data analysis that allows exploring and understanding meaning by engaging research methodologies that honor an inductive style, which means that they focus on individual meaning, and the importance of rendering the complexity of a situation [29].

According to the user interviews, students' responses are blindly hand coded by 3 trained researchers. The codes are grouped under the following themes:

1) Dialog game mobile app is well structured and organized.

2) Dialog game mobile app encourages thinking before response.

3) Dialog game mobile app is engaging and encourages students to speak up their mind in discussions.

4) Dialog game mobile app contributes to a better understanding of other students' perspectives.

5) Dialog game mobile app supports better social interactions compared to traditional methods and online forums.

6) Dialog game mobile app is not suitable for everyday communication.

7) Dialog game mobile app is restrictive (it partially restricts users from spontaneously generating ideas due to interface design).

8) Dialog game mobile app is different from traditional forums or chat applications students use in daily e-learning practices.

\section{RESUlTS AND DisCUSSION}

The usability study showed that users do not spend too much time on execution of user interaction tasks. The two major tasks that took greater execution time are reading instructions and typing into the free-text form. Typing tasks are not independent from users' self-abilities such as typing speed and response speed. Based on the study results, it is difficult to argue whether this part of the design has a lack of usability.

The majority of participants $(80 \%)$ chose to read the instructions before using the main function of the dialog games. Among the rest $20 \%$ only one user return to the home screen to read the instructions later on. This also shows that the design of the mobile app has some frontend design issues i.e. the intuitiveness and the immediate visibility of the purpose of the mobile app. Due to the usability test purposes the research staff did not explain the functions and the purpose of the app, but in real time usage teachers may also explain the educational purposes of the app before students start to use it.

Interview results yielded that, according to the participants, the dialog game mobile app provides a platform which enables students to have an open social interaction and to develop a deeper awareness of other students' perspectives. For example, one of the participants indicated this aspect of the dialog game mobile app as follows: "I may be more open to new ideas and I can accept my friends thought more easily after using the game, as they can easily express their feelings and thoughts"

Similarly, the majority of the students think that the dialog game mobile app allowed them to think in different ways because the app enabled them to see others' mindset and induced them to respond to different topics which may have been initially unfamiliar. It also helped them to think creatively in the discussions: "Since it exposes me to other people's views, it will give me more perspectives, which is crucial for critical thinking, and also creative thinking." or "Discussing controversial topic will make you realize things that you may not actually realize on your daily life, things you often overlook."

Students $(60 \%)$ also indicated that the dialog game mobile app helped them to improve their argumentation skills and to focus and think in a systematic way with the use of structured dialogs and a preset topic. The different types of moves in the game encouraged students to structure their mind and use a variety of responses in their argumentations. Here are some examples from the interviews where students indicate these potentials of the dialog game mobile app: "It can give me a 
more systematic way to present my argument and thus makes my argument stronger. It helps keeping my arguments relevant, sound and succinct." or "Maybe with dialogue games I can be more varied in my responses and attitude toward argumentation skill, not only agree/disagree but also propose and challenge. And many other features that they give as the option, so that we can remember there are other ways/ attitude to respond to an argument."

Some students think that the dialog game mobile app also encouraged them to initiate actions in social interaction. They indicated that the app is especially suitable for those students who are too shy to convey their view in a classroom discussion: "Moreover, you don't have to stand up in the classroom to voice out your opinion, which may be very scary for some students, thus people will not judge you and enable us to be more honest about our thoughts."

Although there are positive potentials of the dialog game mobile app, some students also indicated that they might not use the tool for everyday communication. For example, many students informed that the dialog game app is just suitable for school purposes: “... in daily interaction we don't use these types of tools. We normally talk with each other; we will sit around in a circle and talk about it." or "I think the purpose of this app. maybe for those in schools for example the primary or secondary school students, who need to work in their argumentation skills. The app is not for everyday use."

Overall, participants had no difficulty in terms of executing the tasks using the dialog game mobile app. Although the immediate use and purpose of the dialog games are not obvious in the current design of the mobile app, users had no difficulty in understanding and executing the tasks. In a future design, this issue could be addressed.

In general, the majority of the participants agreed that the app has positive and beneficial potentials to learn and teach effective argumentation skills, critical thinking and creativity skills. Students emphasized that the app has potentials in structuring their thinking, allowing open communication and encouraging social interactions and understanding others' perspectives.

\section{CONCLUSION}

This paper discusses the design of dialog games app for tablet devices. Furthermore, the paper provides the results of the usability test and of the interview analyzing students' attitudes toward the design and use of dialog games on tablet devices.

The results of the usability study indicate that the app is fairly useable. However, there are issues on the immediate intuitiveness of its intended purpose.

The results of the interview show that students have positive attitudes toward the app. They think that the app has positive pedagogical potentials in learning effective argumentation, critical thinking and creativity. Students indicated that the app supported them in structuring their thinking, allowing open communication and encouraging social interactions and understanding others' perspectives.

\section{FUTURE STUDIES}

This study has certain limitations, such as the sample size and data sets. The collected data set is primarily based on qualitative data. Thus, the results may be verified by quantitative studies with a larger sample size in the future. The prototype version of the app may be improved within the light of the current findings. Furthermore, the design can be tested with other PDA devices as well as other mobile operating systems such as Android.

Direct learning outcomes of using a mobile app version of dialog games can also be tested in controlled studies in the future.

\section{ACKNOWLEDGMENTS}

It is my pleasure to express gratitude to Ms. Natalia Darmaja, a research student who contributed much to this project during her participation in A*STAR Student Research Attachment Program.

\section{REFERENCES}

[1] Horizon 2020. The EU Framework programme for research and innovation. [Online]. Available: http://ec.europa.eu/programmes/horizon2020

[2] Partnership for 21st Century Skills. Framework for 21st century learning. [Online]. Available: http://www.p21.org/our-work/p21-framework

[3] A. Ravenscroft, R. Wegerif, and R. Hartley, "Reclaiming thinking: Dialectic, dialogic and learning in the digital age," BJEP Monograph Series II, Number 5-Learning through Digital Technologies, 2007, vol. 1 , no. 1, pp. 39-57.

[4] S. McAlister et al., "Designing to promote improved online educational argumentation: An evaluation study," Networked Learning, 2004, pp. 541-548.

[5] P. McBurney and S. Parsons, "Dialogue games in multi-agent systems," Informal Logic, 2001, vol. 22, no. 3.

[6] Ravenscroft and McAliste, "Designing interaction as a dialogue game: Linking social and conceptual dimensions of the learning process," Interactions in Online Education, pp. 73-88, Routledge London and New York, 2006.

[7] A. Ravenscroft and R. M. Pilkington, "Investigation by design: Developing dialogue models to support reasoning and conceptual change," International Journal of Artificial Intelligence in Education, 2000, vol. 11, no. 1, pp. 273-298.

[8] D. Reisinger. Mobile app revenue set to soar to \$46 billion in 2016. [Online]. Available: http://news.cnet.com/8301-13506_3-57379364-17/mobile-app-revenu e-set-to-soar-to-\$46-billion-in-2016

[9] I. Yengin and B. Lazarevic, "The DiaCog: A prototype tool for visualizing online dialog games," Interactions. Research in Higher Education Journal, 2014, vol. 25.

[10] D. E. Yang. Emerging technologies in higher. [Online]. Available: http://www.foxnews.com/fncu/current-interns/blog/2012/02/21/emerg ing-technologies-higher-ed

[11] S. McAlister et al., "Combining interaction and context design to support collaborative argumentation using a tool for synchronous CMC," Journal of Computer Assisted Learning, 2004, vol. 20, no. 3, pp. 194-204.

[12] A. Ravenscroft and R. M. Pilkington, "Investigation by design: Developing dialogue models to support reasoning and conceptual changem" International Journal of Artificial Intelligence in Education, 2000, vol. 11, no. 1, pp. 273-298.

[13] A. Ravenscroft, "Designing argumentation for conceptual development," Computers \& Education, 2000, vol. 34, no. 3, pp. 241-255.

[14] A. Ravenscroft and M. P. Matheson, "Developing and evaluating dialogue games for collaborative e-learning," Journal of Computer Assisted Learning, 2002, vol. 18, no. 1, pp. 93-101.

[15] A. Ravenscroft et al., "Ambient pedagogies, meaningful learning and social software," Handbook of Research on Social Software and Developing Community Ontologies, 2007, pp. 415-433, Hershey: PA.

[16] A. Ravenscroft, "Promoting thinking and conceptual change with digital dialogue games," Journal of Computer Assisted Learning, 2007, vol. 23 , no. 6 , pp. $453-465$. 
[17] E. F. Barkley et al., Collaborative Learning Techniques: A Handbook for College Faculty, John Wiley \& Sons, 2014.

[18] C. Dede, "Comparing frameworks for 21 st century skills. 21st century skills: Rethinking how students learn," pp. 51-76, 2010.

[19] I. Yengin and B. Lazarevic, "The DiaCog: A prototype tool for visualizing online dialog games' interactions," Research in Higher Education, 2014.

[20] R. Wegerif, "A dialogic understanding of the relationship between CSCL and teaching thinking skills," International Journal of Computer-Supported Collaborative Learning, 2006, vol. 1, no. 1, pp. 143-157.

[21] W. Turnbull and J. I. M. Carpendale, "Talk and the development of social understanding," Early Education and Development, 2001, vol 12, no. 3, pp. 455-478.

[22] S. Feller and I. Yengin, "Guidelines for the design of dialogic educational technology," Educating in Dialog: Constructing Meaning and Building Knowledge with Dialogic Technology, 2014, vol. 24, p. 223.

[23] J. Dillon, "Discussion, debate and dialog: Changing minds about conceptual change research in science education," Cultural Studies of Science Education, 2008, vol. 3, no. 2, pp. 397-416.

[24] A. Ravenscroft et al., "Reclaiming thinking: dialectic, dialogic and learning in the digital age," Number 5-Learning through Digital Technologies, 2007, vol. 1, no. 1, pp. 39-57.

[25] Pc World. Review: LiveCode community is open-source hypercard for the 21st century. [Online]. Available: http://www.pcworld.com/article/2040331/review-livecode-community -is-open-source-hypercard-for-the-21st-century.html

[26] J. Creswell, Qualitative Inquiry and Research Design: Choosing Among Five Traditions, 3rd ed. Thousand Oaks, CA: Sage, 2008.

[27] J. W. Creswell, Research Design Qualitative, Quantitative and Mixed Method Approaches, Thousand Oaks, CA: Sage, 2009.

[28] G. R. Gibbs, Analyzing Qualitative Data, London: Sage, 2007.
[29] J. S. Dumas, "Usability testing methods: Think-aloud protocols," Design by People For People: Essays on Usability, UPA, 2001, pp 119-129.

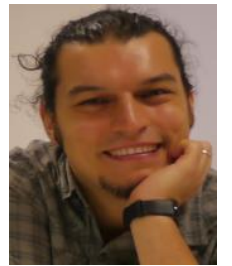

Ilker Yengin is a researcher at the Institute of High Performance Computing of Agency of Science, Technology and Research (A*STAR), Singapore. He is the co-founder of "Collaborative Thinking and Technologies" (CTT) research group and leads educational technology projects at the "Intelligent Social Educational Technologies" (iSET) program. With a background in Educational Technologies and Human-Computer Interactions (HCI), Yengin brings design- based research solutions to the problems of collaborative learning, thinking and creating in a social setting. Dr. Yengin specialized in the design of interactions with technologies which enable collaborative learning, thinking and creating. These technologies allow co-creation of knowledge, ideas and perceptions in social settings. His research provides an understanding of the design and role of these technologies on different topics such as human cognition, learning, social interaction, concepts of identity and positive emotions (motivation).

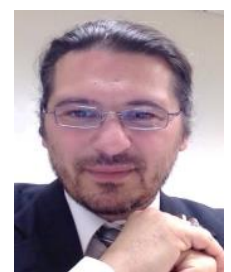

Bojan Lazarevic received a doctoral degree in education, Instructional Technology from the University of Nebraska, while his background is in pedagogy and adult education with a teaching methods emphasis. He specializes in areas of e-learning, Web 2.0, instructional design, emerging technologies and production of multimedia learning materials. Dr. Lazarevic is an associate professor in the Department of Mathematics and Informational Technology responsible for leading the Educational Technology Program at Mount Saint Mary College. Prior to the Mount, he taught at the University of Belgrade, Republic of Serbia for seven years and Instructional Technology courses for pre-service teachers (K-12) at the University of Nebraska-Lincoln for four years. 